\title{
Arduino Based Forward Collision Detection Warning System
}

\author{
Orji E.Z ${ }^{1}$, Oleka C.V ${ }^{2}$, and Nduanya U.I ${ }^{3}$ \\ Computer Engineering Department, Enugu State University of Science and Technology, Enugu, Nigeria ${ }^{1,2,3}$
}

\begin{abstract}
Most new cars today come with a host of advanced safety features including automated systems that assist the drive in maintaining control of the car and warning the driver of possible dangers. The problem, however, is that while these kinds of features greatly increase the safety of a car, they are exorbitantly expensive and only available in new, high-end cars not in old, low-end cars. This research paper aim at creating forward collision mitigation system that provide a level playing ground for all vehicles, regardless of make, age, type or model, when it comes to possible safety risk one can face on the road and at a minimal cost. The system will detect the distance between two vehicles moving in the same lane, in the same direction and alert the driver whenever he or she is in danger range using a microcontroller and ultrasonic sensor. The distance is measured by an ultrasonic and an audio visual alarm to effectively alert the driver and/or the passengers.
\end{abstract}

Keywords: Collision, Sensor, Cars, Arduino, Accident, Vehicle, Ultrasonic, Alert, Risks

\section{INTRODUCTION}

The automotive industry of modern days are far from been defined by expensive cars, flashy cars, sports cars and big muscle cars. Rather, most of the cars seen on the roads today are dominated by features such as safety systems and how well the car can withstand accidents without injuring the driver and passengers within. Automobile manufacturers today can effortlessly assemble extremely safe cars for anybody willing to pay top bill, with some of these safety features now standard making them extremely expensive. As long as cars remain on the road safety is a top priority for automobile manufacturers and will continue to be for a very long time.

Question one would ask is what about old generation cars? Generation when 4WD and heavy duty engine cars are more appealing than cruise control, automatic transmission, or navigation system found in modern cars. For those cars, certain amount of safety risk is assumed when they are on the road, but not enough risk that will necessitate their removal from the road by the authority. They are assumed to be adequately safe but cannot measure up with safest cars of 2018[1] like Lexus, Mazda, and Acura with their multitude of sensors, and high-intensity discharge (HID) lights, how safe can they really be on the road?

This research paper aim at creating Arduino based forward collision mitigation system that provide a level playing ground for all vehicles, regardless of make, age, type or model, when it comes to possible safety risk one can face on the road and at a minimal cost. In other to have a successful system, it needs to addresses two key issues, cost and flexibility. Firstly, the system must be cost-effective but provide a certain level of safety expected from device of similar services. Secondly, the system also needs to be flexible enough to cover wide range of cars, which could span any number of years to modern day cars.

\section{LITERATURE REVIEW}

Wenjie Song et al [2] present a real-time and robust lane detection and forward collision warning technique based on stereo cameras. Obstacles image is obtained through stereo matching and UV-disparity segmentation algorithm. Then, Inverse Perspective Mapping (IPM) and Sobel filtering are conducted to generate a low-noise top view of the road by fusing the obstacles image and the original image. Next, Hough Transformation for the top view map is completed and the extreme points (poles) are calculated as the detected lanes according to the traffic lanes model. The host lane is selected or supplemented among all the detected lanes and the nearest obstacle in this host lane is detected for the forward collision warning. Experimental results on the public data set indicate that the author's method can work 
International Journal of Advanced Research in Computer and Communication Engineering

\author{
Vol. 7, Issue 9, September 2018
}

effectively and in real-time in the normal structured environment. However, the system is sometimes influenced by the bad factors like railway tracks light reflecting, or dazzling light on the road which can lead to false detection of lane samples.

Author Narayan Srinivasa [3] proposed vision-based vehicle detection and tracking method for forward collision warning in automobiles. The approach used is based on a set of edge-based constraint filters that assist in the segmentation of vehicles from background clutter. The detected vehicles are then tracked using a combination of distance based marching, sum-of square-of-difference in intensity (SSD) and edge density of detected vehicle regions. The computational load for tracking is minimized using a vehicle-clustering algorithm that enables the tracking of nearby vehicles while ignoring far away vehicles. The system works well since the algorithm does not rely on lane tracking for processing, and it can complement any forward collision warning system for threat assessment. Since the system uses camera in its edge detection, bad weather can induce noise which leads to significant number of false negatives.

Our method includes an ultrasonic sensor, which can detect a vehicle in front of the host car and alert the driver to the danger through an auditory warning. Also integrated in the system is an LED array, which could equally alert the driver about the proximity of the host car to the one in front. The system relies on communication with the ultrasonic sensor to obtain a reading of proximity from other cars, and in turn switches the LEDs from their steady-burn state to a flashing pattern, warning host driver of the danger ahead.

\title{
3. SYSTEM DESIGN
}

Our basic approach for Arduino based forward collision detection warning system to integrate obstacle detector using ultrasonic sensor and audio/visual warning subsystem using LEDs and buzzer to alert the driver when the vehicle is in verge of collision. In distance between the host vehicle and leading vehicle is large $(>50 \mathrm{~cm})$ the host vehicle is in safe distance, when the distance is less $(<=50 \& \&>=20 \mathrm{~cm})$, visual alert is used to warn the driver, but when the distance is much less $(<20)$ an action is needed from the driver, so visual and audio alert is used in warning the driver to avoid collision.

The safe distance model in this paper is based on the critical warring distance proposed by Nakaoka [4] and also used by Woon-Sung [5]. The equation (1) descried the safe distance Ds on the system.

$$
D_{s}=\frac{v^{2}}{2_{a}}-\frac{\left(V_{f}-V_{r}\right)^{2}}{2\left(a-a_{r}\right)}+D_{m}
$$

Where: $V_{f}$ is the speed of the following vehicle, $V_{r}$ is the difference in speed between following and the leading vehicles, $a_{r}$ is the difference in acceleration between the following and the leading vehicles, and $D_{m}$ safety margin distance when both vehicles.

Relative speed $\Delta v$ [6] between the following vehicle and the leading vehicle of the system can be calculated using the following formula as seen in equation (2).

$$
\Delta v=v_{l}-v_{f}
$$

Where: $v_{l}$ is the speed of the leading vehicle and $v_{f}$ is the speed of the following vehicle.

The Arduino based forward collision detection warning system can react in three ways, as illustrated in figure 1:

First scenario, if the relative distance between the following and the leading vehicle is larger than the safe distance $(>50 \mathrm{~cm})$, the following vehicle is in safe distance hence, no action required.

Second scenario, if the relative distance becomes smaller than the safe distance $(50 \mathrm{~cm})$ and larger or equal to $20 \mathrm{~cm}$, visual alert only is activated by the system signaling the driver to maintain safe distance.

Third and final scenario, if the relative distance becomes smaller than $20 \mathrm{~cm}$, visual and audio alert of the system is activated indicating that the vehicle is in verge of collision. 
Vol. 7, Issue 9, September 2018
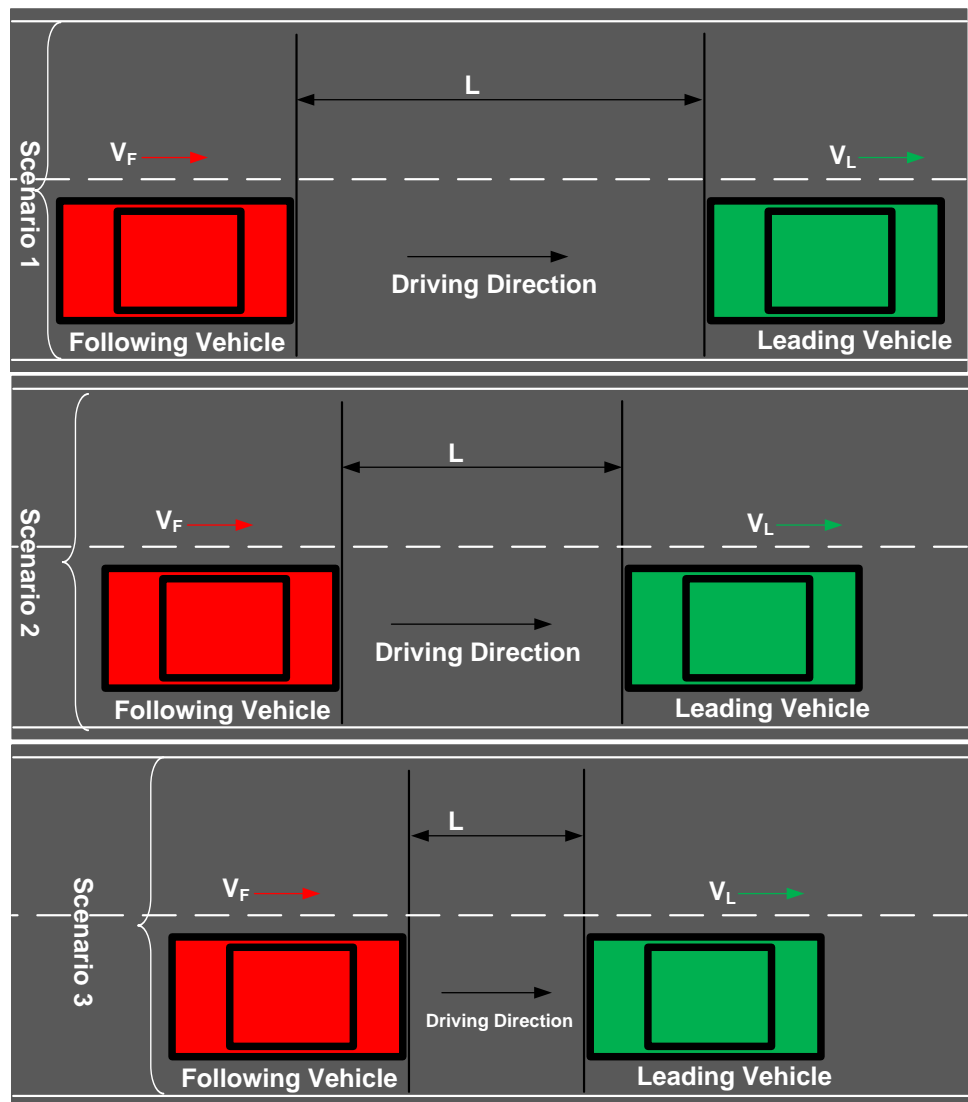

Figure 1: Proposed approach for collision detection and warning system

\subsection{System Block Diagram}

The Arduino based forward collision detection warning system designed in this paper consists of hardware and software part. The hardware consists of the system circuit while the software part deals with the programming of the microcontroller using Arduino IDE.

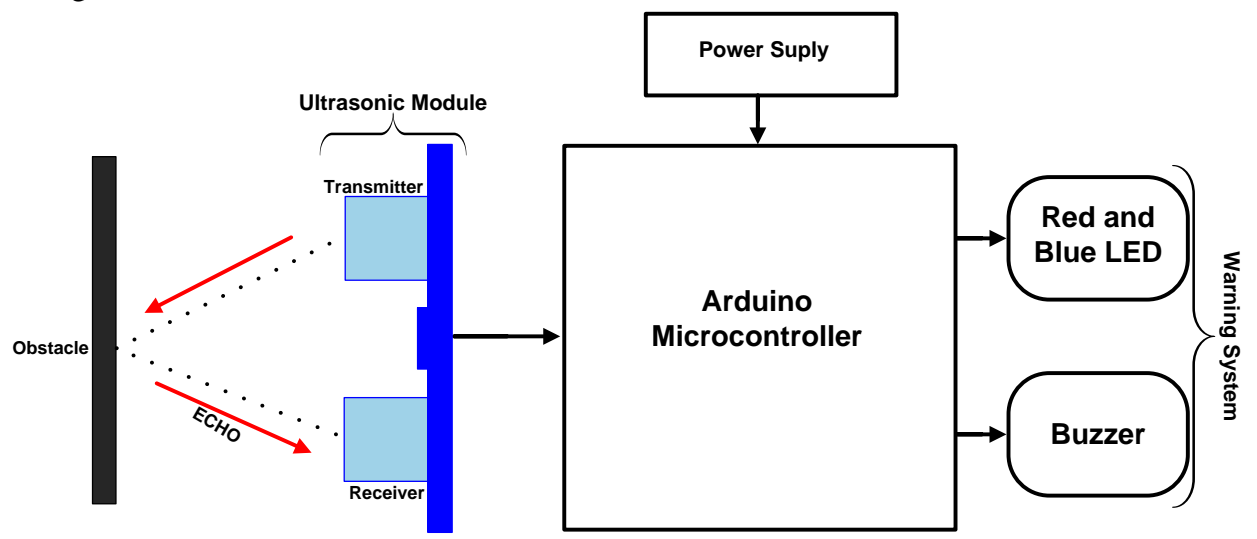

Figure 2: System Block Diagram

The system block diagram in figure 2 consists of four sub-systems; the power supply, the microcontroller, ultrasonic module, and the warning system.

Warning Unit - This subsystem provides reliable warning to driver about a possible collision prior to their reaching an unsafe position or location. The composition of the warning system comprises of the buzzer and LED. The warning subsystem has three operational modes. First, when there is no vehicle detected in the range $(>50 \mathrm{~cm})$ of the host vehicle, no action is required. Second, a red LED will light upon detecting a vehicle and the distance between both vehicles is $\langle=50 \mathrm{~cm}$ and $>=20 \mathrm{~cm}$. The last mode is when a vehicle is detected and the distance between both vehicles is $<20 \mathrm{~cm}$, visual (blue LED) and audio (buzzer) is triggered. 
Vol. 7, Issue 9, September 2018

Ultrasonic Module - In the project, we used ultrasonic sensor which is an electronic device that emit acoustic wave beyond audible range, between $20 \mathrm{~Hz}$ to $20,000 \mathrm{~Hz}$ to determine the distance between sensor and object based on the time it takes to send the signal and to receive the echo.

\subsection{System Control Flow}

Figure 3 is the system flow chart. The Arduino microcontroller receives ECHO signal from the ultrasonic sensor. This information received from the ultrasonic sensor is used to excite solenoids to create electromagnetic field. The ultrasonic sensor continuously read distance between the leading vehicle and following vehicle.

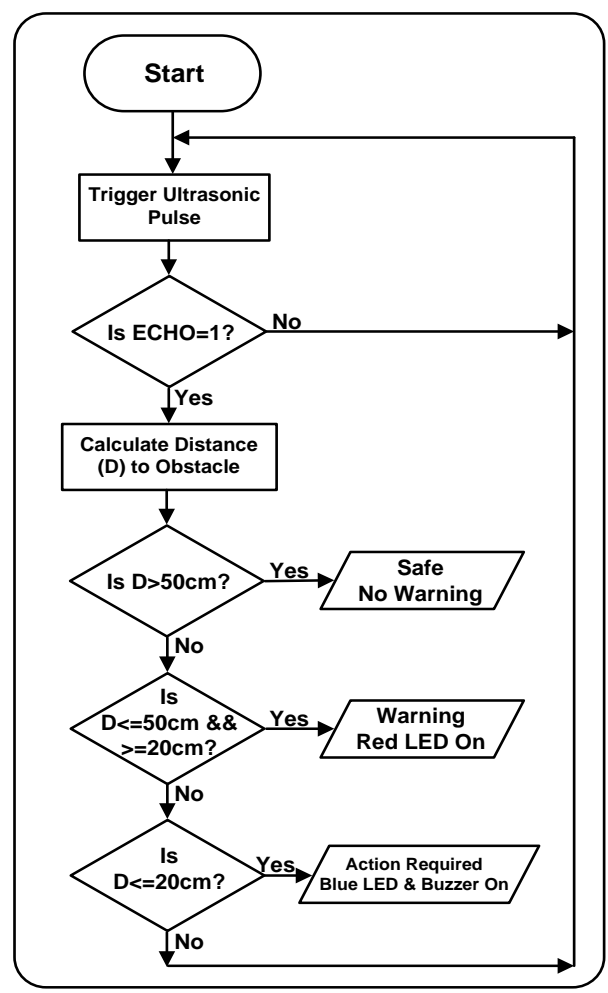

Figure 3: System Flow Chart

If the ultrasonic sensor detects a vehicle, it calculates the distance between the two vehicles. If the distance is $>50 \mathrm{~cm}$, the following vehicle is in safe distance, no warning is needed but if the distance between the two vehicle is $<=50 \mathrm{~cm}$ $\& \&>=20 \mathrm{~cm}$, the visual alert is activated. The last state is when the distance between both vehicles is $<20 \mathrm{~cm}$, visual and audio alert is activated signaling to the driver that the vehicle is in verge of collision.

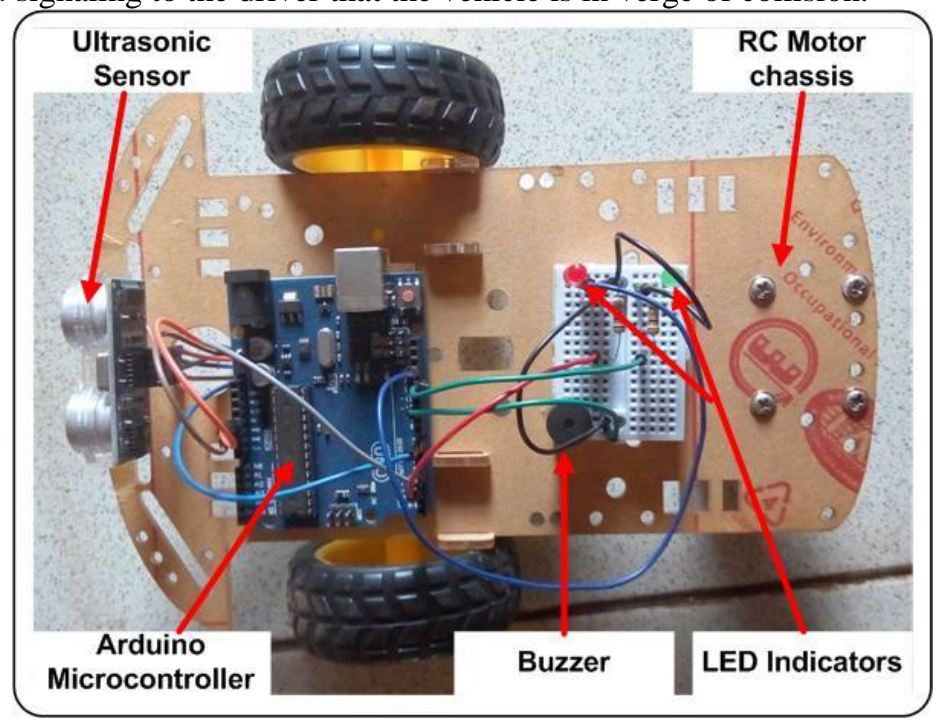

Figure 4: Arduino Based Forward Collision Detection Warning System 
Vol. 7, Issue 9, September 2018

Our system was mounted on an RC motor chassis as shown in figure 4, it was also tested for vehicle detection. The system detected objects and appropriate alert give, no alert if the object in more than $50 \mathrm{~cm}$ away, flashing of red LED if it is less than $50 \mathrm{~cm}$ but more than $20 \mathrm{~cm}$ and lastly, blue LED and buzzer if the object is less than $20 \mathrm{~cm}$ to the host vehicle.

\section{DISCUSSION OF RESULT}

Distance between following and leading vehicle is very important in this project that is why our testing is based on that factor. Also various system subsystems were also testing to make sure their connection is firm and give desired result. Collision Distance: The distance measured by the ultrasonic sensor were found to vary from the actual measurement taken, since the reliability of the system is very much dependent on the distance acquired from the sensors it was calibrated to give the expected readings. Distance results are shown in Table 1.

Table 1: Collision Distance Testing

\begin{tabular}{c|c|c}
\hline Test & Expected Distance $(\mathbf{c m})$ & Measured Distance $(\mathbf{c m})$ \\
\hline 1 & 10 & 7 \\
2 & 20 & 19 \\
3 & 30 & 25 \\
4 & 40 & 38 \\
5 & 50 & 47 \\
\hline
\end{tabular}

From table 1, expected distance and measured distance defers in all five tests of various distances. So percentage error (PE) is calculated using equation (3) [7] to determine how much error in the system.

$$
P E=\frac{E_{A v g}-M_{A v g}}{M_{A v g}} \times 100
$$

Where: $E_{A v g}$ and $M_{A v g}$ is expected distance and measured distance average respectively. The percentage error of the system is $10.1 \%$.

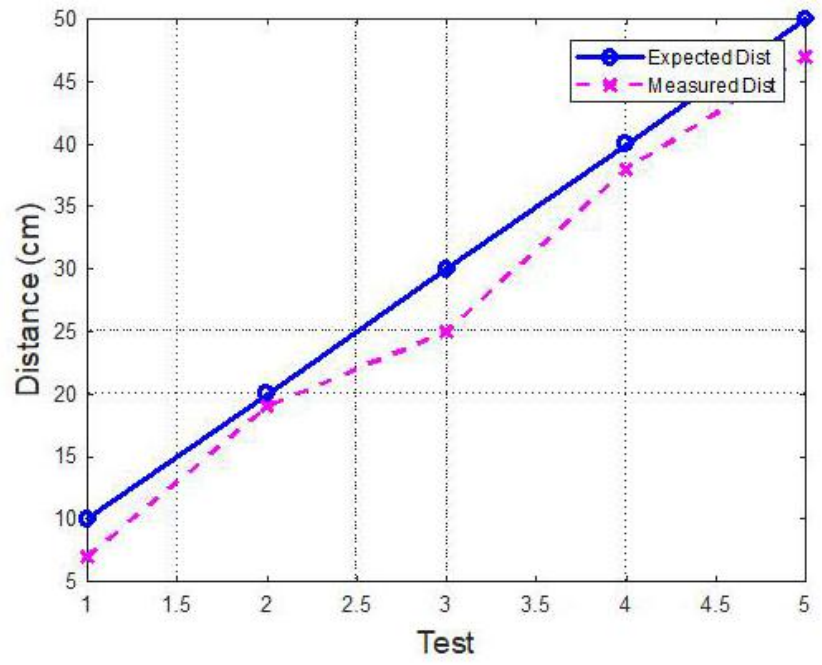

Figure 5: Distance against Test of Expected and Measured Distance of the system

From the graph in figure 5 it can be seen that in all five tests carried out the difference between the expected and measure distance is not much and average of $2.8 \mathrm{~cm}$. It shows that the performance of the system is above average and at a safe distance.

\section{CONCLUSION}

Arduino based collision detection and warning system is designed and mounted on an RC car to demonstrate the system and it was found functional. The ultrasonic sensor was able to read distances of shorter range accurately and alert the driver if the car is in danger of collision. There were differences in the expected distance and measure distance but the system is safe. A distance sensor capable of detecting long distance is recommended for this to be applied in real vehicles. With the right materials, it is possible to improve the system features so that is can be integrated in real vehicles. 
Vol. 7, Issue 9, September 2018

\section{REFERENCES}

[1] Steven Loveday, (2018), "17 Safest Cars of 2018" Retrieved: August 02, 2018, Available at: https://cars.usnews.com/cars-trucks/safest-cars-ofthe-year

[2] Wenjie Song, Mengyin Fu1, Yi Yang, Meiling Wang, Xinyu Wang and Alain Kornhauser, "Real-time lane detection and forward collision warning system based on stereo vision", 2017 IEEE Intelligent Vehicles Symposium (IV), pp. 493-498, June 2017, Redondo Beach, CA, USA

[3] Narayan Srinivasa "Vision-based vehicle detection and tracking method for forward collision warning in automobiles", IEEE Intelligent Vehicle Symposium, 626-631, 17-21 June 2002, Versailles, France.

[4] Nakaoka, M., Raksincharoensak, P., \& Nagai, M. (2008). "Study on forward collision warning system adapted to driver characteristics and road environment", Proceedings of International Conference on Control, Automation and Systems. Korea.

[5] Woon-Sung Lee, Ji-Yong Lee, and Sang-Soo Park (2011), "A new approach to forward collision avoidance", 3rd International Conference on Road Safety and Simulation, Indianapolis Indiana, United States.

[6] Wenshuo Wang, Student Member, IEEE, Junqiang Xi, and Ding Zhao, "Learning and Inferring a Driver's Braking Action in Car-Following Scenarios", IEEE Transactions on Vehicular Technology January 2018.

[7] Anne Marie Helmenstine, Ph.D., (2018), "How to Calculate Percent Error" Retrieved: September 11, 2018, Available at: https://www.thoughtco.com/how-to-calculate-percent-error-609584 